\title{
Changes in the prevalence, incidence and residual risk for HIV and hepatitis $C$ virus in Southern Brazilian blood donors since the implementation of NAT screening
}

\author{
Emil Kupek ${ }^{[1]}$ and Andrea Petry ${ }^{[2]}$
}

[1]. Departamento de Saúde Pública, Universidade Federal de Santa Catarina, Florianópolis, SC. [2]. Centro de Hematologia e Hemoterapia do Estado de Santa Catarina, Florianópolis, SC.

\begin{abstract}
Introduction: Previous studies have shown high residual risk of transfusing a blood donation contaminated by human immunodeficiency virus (HIV) or hepatitis $\mathrm{C}$ virus (HCV) in Brazil and motivated the development of a Brazilian platform for simultaneous detection of both viruses by nucleic acid amplification test (NAT) denominated HIV/HCV Bio-Manguinhos/ Fundação Oswaldo Cruz (FIOCRUZ). The objective of this study was to verify seroprevalence, incidence and residual risk for both viruses before and after the implementation of NAT. Methods: Over 700,000 blood samples from all blood banks in the southern Brazilian State of Santa Catarina were analyzed during the period between January 2007 and July 2013 . Results: Compared with the period preceding the NAT screening, HIV prevalence increased from 1.38 to 1.58 per 1,000 donors, HIV incidence rate increased from 1.22 to 1.35 per 1,000 donor-years, and HIV residual risk dropped almost 2.5 times during the NAT period. For HCV, seroprevalence increased from 1.22 to 1.35 per 1,000 donors, incidence dropped from 0.12 to 0.06 per 1,000 donor-years, and residual risk decreased more than 3 times after the NAT implementation. Conclusions: NAT reduced the duration of the immunologic window for HIV and $\mathrm{HCV}$, thus corresponding to approximately 2.5- and 3-fold respective residual risk reductions.
\end{abstract}

Keywords: HIV. HCV. Blood donors. Prevalence. Incidence. Residual risk.

\section{INTRODUCTION}

Blood safety in transfusion medicine critically depends on donor recruitment, clinical examination for signs and symptoms of past or present infections and exclusion of those who represent a risk of transmitting these infections to the blood recipients; this screening relies either on pre-donation interviews or laboratory screening via serological or nucleic acid amplification test (NAT) analyses ${ }^{1}$. However, despite an increase in sensitivity with this screening, there is still some residual risk that the donated blood may be contaminated by an infectious agent. The quantification of residual risk is an important part of blood safety policies worldwide ${ }^{2}$. In Brazil, the methodology of residual risk estimation had been disseminated in the Portuguese language by the end of the $1990 \mathrm{~s}^{3,4}$ and has been sporadically applied in the country since then ${ }^{1,5-10}$. The results of these studies showed

Address to: Dr. Emil Kupek. Deptō Saúde Pública/UFSC. Campus Universitário Trindade, 88040-900 Florianópolis, SC, Brasil.

Phone: 5548 3721-9388

e-mail: emil.kupek@ufsc.br

Received 6 June 2014

Accepted 11 August 2014 high prevalence, incidence and residual risk in Brazil compared with the United States of America (USA), Europe, Japan and Australia, thus reinforcing the need to monitor these parameters in a systematic way and to use them to evaluate the effectiveness of transfusion safety measures.

By the end of 2010, the Brazilian Ministry of Health had started the development of a NAT platform for the simultaneous detection of human immunodeficiency virus (HIV) and hepatitis $\mathrm{C}$ virus (HCV). After piloting the platform in three blood banks in 2010, the Brazilian NAT kit was licensed for routine blood bank screening in 2011 and has since been gradually implemented throughout the country. Two window-period transmissions of HIV were reported during the piloting phase, both of them in $2009^{11}$.

The objective of this paper is to compare the prevalence, incidence and residual risk for HIV and HCV before and after the implementation of the Brazilian NAT platform by the Centre for Hematology and Hemotherapy of the Santa Catarina State (HEMOSC - Centro de Hematologia e Hemoterapia do Estado de Santa Catarina) in July 2010.

\section{METHODS}

The study population included all HEMOSC blood donors eligible for blood screening after clinical examinations and pre-donation interviews between January 2007 and July 2013. 
HEMOSC is a network of six blood banks from the Cities of Blumenau, Chapecó, Criciúma, Joaçaba, Joinville and Lages that provides $99.5 \%$ of the blood and hemocomponents transfused in the State of Santa Catarina of approximately six million inhabitants. It was certified with the ISO9001: 2010 quality control stamp. Since 2007, all blood screening has been performed in the central laboratory in the state capital of Florianópolis.

Data were extracted from the HEMOSC computerized donor records and included demographic information (age, sex, location of residence); laboratory test results for HIV, $\mathrm{HCV}$, human T lymphotropic virus (HTLV), hepatitis B virus (HBV), T. cruzi and T. pallidum; and details regarding previous donations (dates, test results, impediments). The anti-HIV-1,2 $\mathrm{O}$ Prism and anti-HCV Prism tests, both manufactured by Abbott Laboratories (Wiesbaden, Germany), were used for HIV and HCV serological screening. In addition, a fourthgeneration antigen/antibody kit, the Enzygnost Integral II (Siemens, Marburg, Germany), was used between January 2007 and July 2010 before NAT screening for HIV and HCV was introduced. Along with the NAT implementation, the combined antigen/antibody kit for HIV was substituted with the anti-HIV Combo test (Architect Laboratories, Wiesbaden, Germany). The Brazilian duplex HIV/HCV NAT platform was developed and supplied by Bio-Manguinhos/Fundação Oswaldo Cruz (FIOCRUZ, Rio de Janeiro, Brazil) as a six-sample mini-pool. All NAT-screen-positive samples were also tested by INNO-LIA immunoblot (Innogenetics, Gent, Belgium).

An exact Poisson distribution was used to determine the 95\% confidence interval (CI) for the number of HIV- and $\mathrm{HCV}$-positive samples. For repeat donors, the HIV and HCV seroconversion date was estimated as a mid-point between the last negative donation and the first positive test result ${ }^{12}$. Residual risk was calculated by the incidence/window method, which multiplies the probability of viral conversion during the interdonation interval by the immunologic window duration ${ }^{12}$. The probability was calculated as the conversion incidence rate, which divides the number of converting repeat donors by their time at risk. The latter was estimated by summing the interdonation intervals between the last two donations for the screennegative donors and half the intervals for the screen-positive donors. This calculation assumes that the risk of conversion is equally spread over the intervals, so that their mid-point is the best unbiased estimate on the group level.

Based on the kit manuals provided by their manufacturers, the window durations were as follows: 22 days for the Prism anti-HIV test, 32.8 days for the Prism anti-HCV test, 17 days for the Enzygnost Integral II test, 14 days for the HIV Combo Architect test and 9 days for both HIV and HCV NAT screening.

Stata software, version 11.0 (StataCorp, College Station, TX, USA), was used for all statistical calculations.

\section{RESULTS}

During the period analyzed, 293,725 blood donors provided 719,223 blood samples that were screened for the aforementioned infectious agents. Before and after the NAT was implemented in HEMOSC, there were 168,318 and 125,378 blood donors, respectively, whose blood samples were screened. The number of samples includes repeated tests for screenpositive test results.

The blood donor profile was somewhat altered after the NAT implementation. The participation of first-time donors increased from $69.7 \%$ to $91.6 \%$, as did that of women (from $38.1 \%$ to $44.6 \%$ ) and of donors from Blumenau, where blood bank expansion took place after the NAT screening was introduced. The blood donors whose previous donations occurred within 12 months of their last donation decreased from $10.1 \%$ to $0.6 \%$, whereas those with longer inter-donation intervals decreased from $20.2 \%$ to $7.8 \%$ of all donors. In contrast, there was an increase in the recruitment of blood donors from the youngest (16-24 years) age group. Donor race, educational level and prevalence of co-infections (i.e., infections other than HIV or HCV) remained roughly the same before and after the NAT was introduced.

HIV prevalence rates per thousand donors were 1.38 and 1.58 before and after NAT implementation, respectively (Table 1). The repeat donors had a significantly higher prevalence than the first-time donors, particularly those with shorter inter-donation intervals. The HIV prevalence was twice that in male donors compared with female donors. Older age, lower educational level and co-infection (mainly with HBV) all increased the likelihood of an HIV-positive test result. Significantly higher HIV prevalence rates were observed for the donors from Florianópolis, Criciúma and Joinville compared with those from Blumenau, Chapecó and Joaçaba, with the City of Lages holding an intermediary position.

HCV prevalence rates per thousand donors were 1.22 and 1.35 before and after NAT implementation, respectively (Table 2). The first-time donors had a significantly higher prevalence than the repeat donors, as did men compared with women. The donors from Florianópolis and Criciúma showed significantly elevated HCV prevalence rates compared with those from Blumenau, Joaçaba and Joinville. There were clear increases in prevalence in older donors, those with lower educational levels and those with co-infections.

The risk factors associated with incidence showed similar magnitudes and directions for both HIV and HCV. HIV incidence was more than ten times higher in the repeat donors with shorter ( $<12$ months) inter-donation intervals compared to those with longer intervals. Male donors had twice the incidence of female donors. The donors between 25 and 34 years of age showed the highest incidence, as did those residing in Criciúma and those with co-infections.

A sharp increase in HIV prevalence among male donors aged 16-24 years was noted (Figure 1). A similar trend was observed for young women until 2012, after which a sharp decline in prevalence was observed (Figure 2).

Residual risk was calculated as the product of the incidence of viral conversion and the duration of the immunological window period (Table 3). For example, the 17-day window for HIV serological tests before NAT implementation is equivalent to 0.0465 years; when multiplied by the HIV-conversion 
TABLE 1 - HIV prevalence and incidence by risk factors.

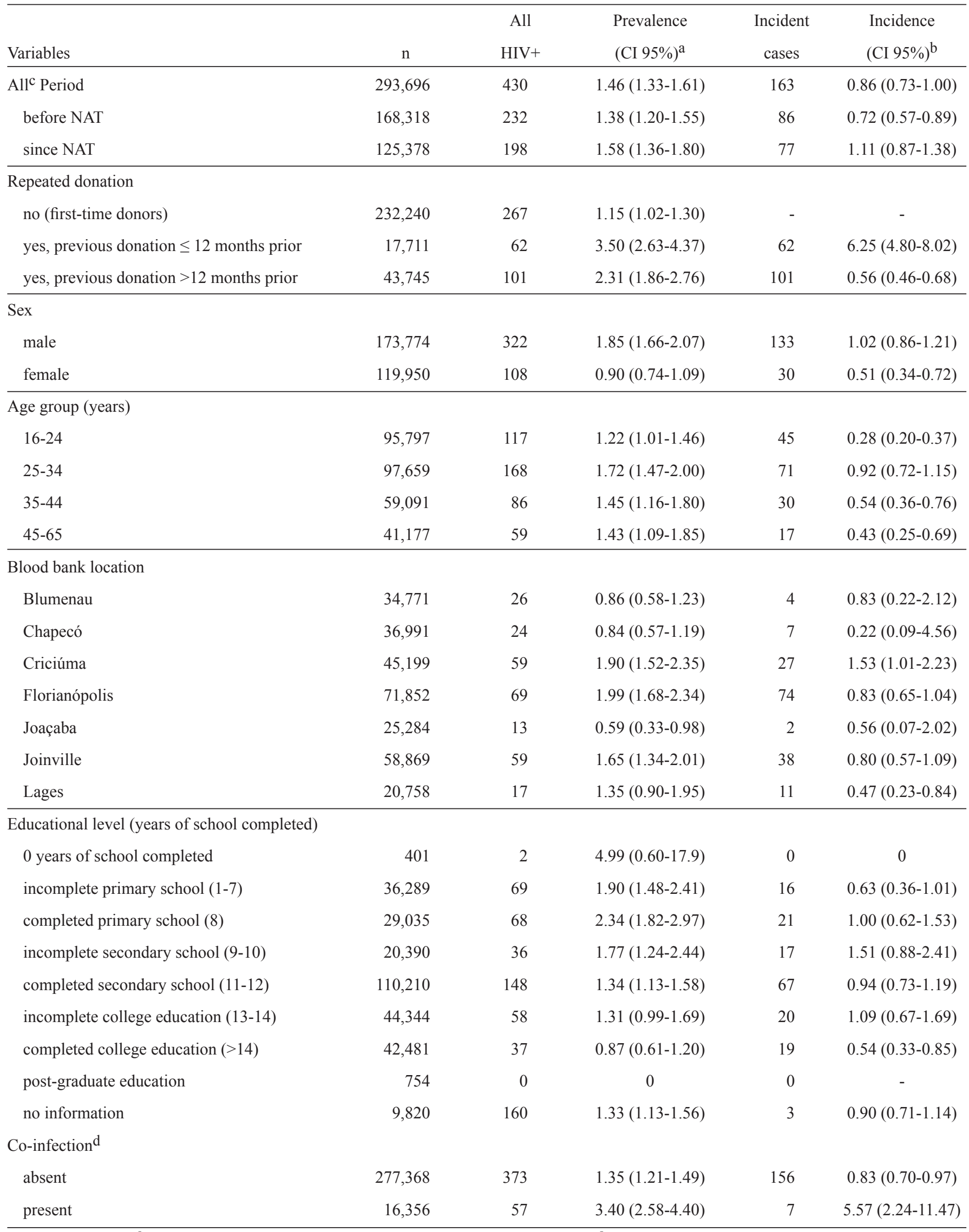

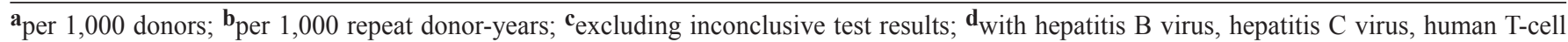
lymphotropic virus, Tryapanosoma pallidum or Tryapanosoma cruzi. HIV: human immunodeficiency virus; NAT: nucleic acid amplification test. 
TABLE 2 - HCV prevalence and incidence by risk factors.

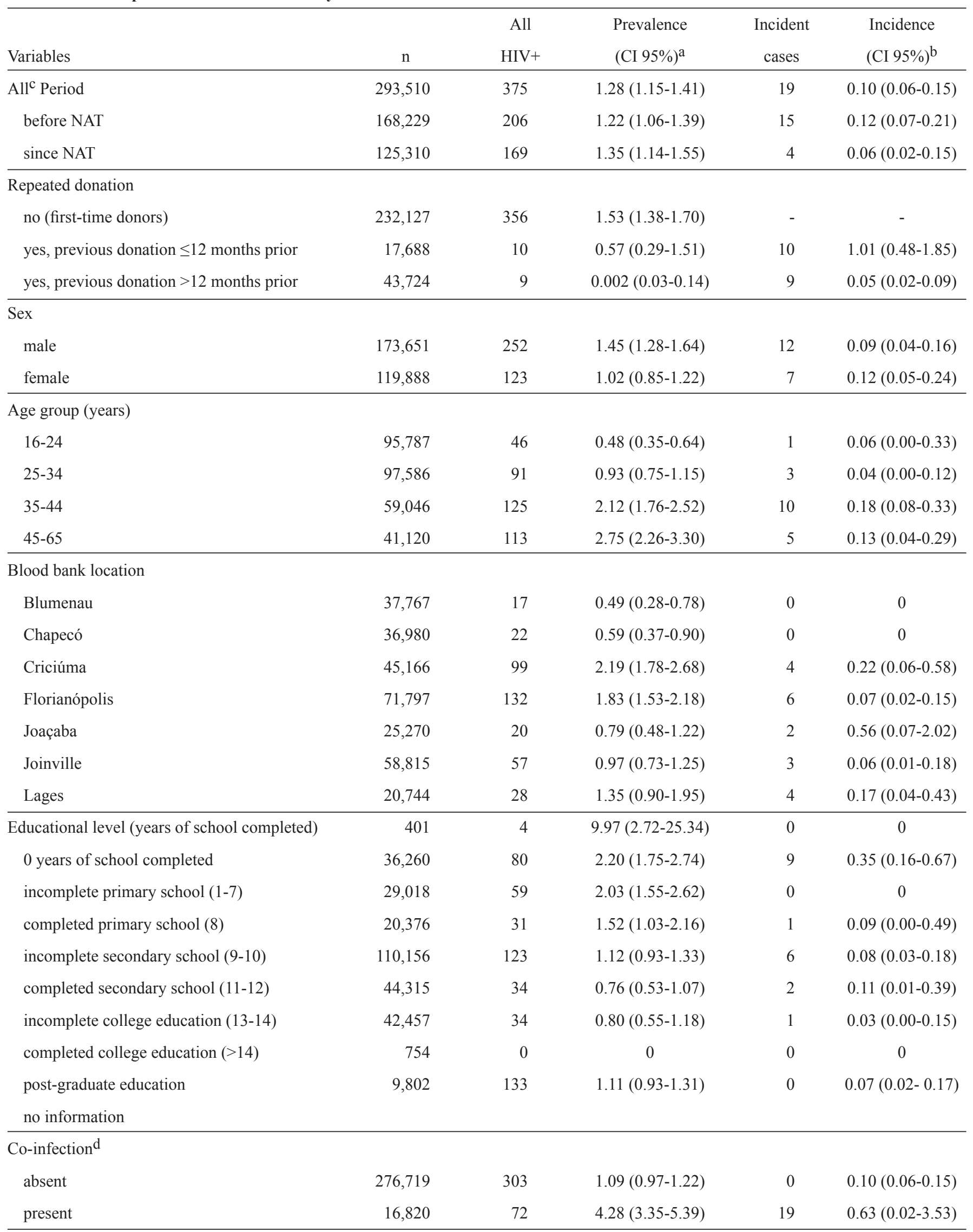

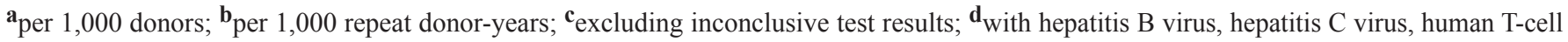
lymphotropic virus, Tryapanosoma pallidum or Tryapanosoma cruzi. HCV: hepatitis C virus; NAT: nucleic acid amplification test. 


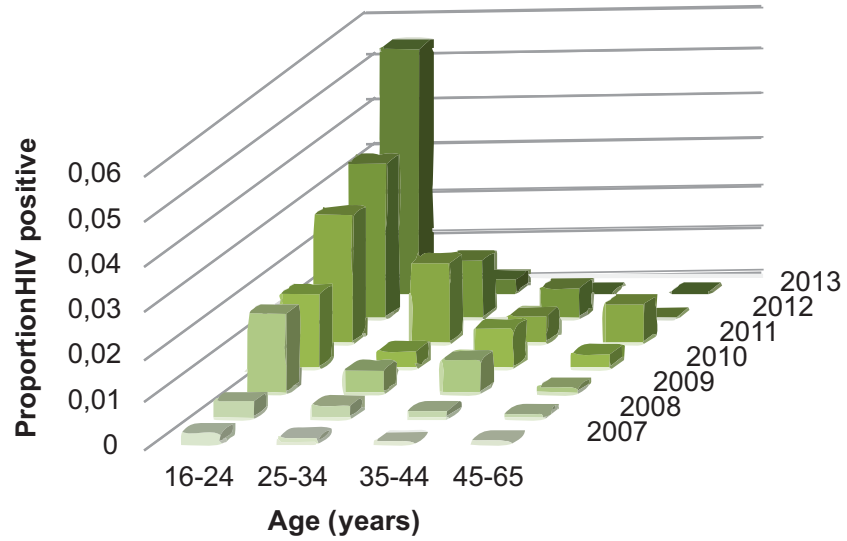

FIGURE 1 - Proportion of male repeat donors infected by HIV, by age group. HIV: human immunodeficiency virus.

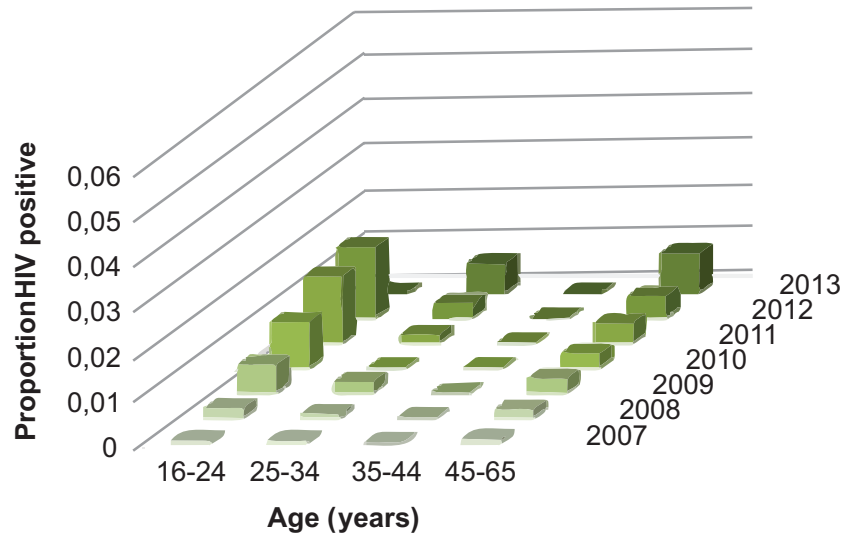

FIGURE 2 - Proportion of female repeat donors infected by HIV, by age group. HIV: human immunodeficiency virus.

incidence of 1.91 per 1,000 person-years in repeat donors with inter-donation intervals of $\leq 12$ months, the residual risk is 8.89 per 100,000 person-years. Since the NAT screening was introduced, the same group reduced both its window period (from 17 to 9 days, or 0.0246 years) and its incidence (from 1.91 to 1.80 per 1,000 person-years), thus producing an HIV residual risk of 4.44 per 100,000 person-years, which is a two-fold reduction compared with the period when only serological screening was conducted. For repeat donors with inter-donation intervals of $>12$ months, the HIV residual risk was reduced 2.62 times. During the NAT screening period, HCV residual risk was reduced 4.26 and 4.53 times for repeat donors with shorter and longer inter-donation intervals, respectively.

Overall, HIV incidence and prevalence increased by $54.2 \%$ (from 0.72 to 1.11 per 1,000 person-years) and $14.5 \%$ (from 1.38 to 1.58 per 1,000), respectively, after NAT implementation, but the differences did not reach statistical significance (Table 1). However, the incidence of HIV conversion in all repeat donors decreased by $24.1 \%$, from 0.54 to 0.41 per 1,000 person-years, thus contributing to the residual risk reduction. The magnitude of the HIV window reduction was even larger, decreasing by $47.1 \%$ (from 17 to 9 days). In contrast, HIV residual risk in all repeat donors decreased 2.5 times after NAT implementation, from 2.51 to 1.01 per 100,000 person-years (Table 3).

Overall, HCV incidence and prevalence increased by $100 \%$ (from 0.06 to 0.12 per 1,000 person-years) and $10.6 \%$ (from 1.22 to 1.35 per 1,000 ), respectively, after NAT implementation (Table 2), but the increases were not statistically significant. Although the incidence of $\mathrm{HCV}$ conversion in all repeat donors increased by $18.5 \%$ (from 0.54 to 0.64 per 1,000 person-years) after NAT implementation, $\mathrm{HCV}$ residual risk during the NAT screening period still decreased approximately threefold compared with the period using only serologic screening (Table 3). This risk reduction was likely driven by a large HCV window reduction of $72.4 \%$ (from 32.6 to 9 days) in the NAT screening period.

\section{DISCUSSION}

Increased participation by first-time donors (from $70 \%$ to over 90\%) and women (from 38\% to almost $45 \%$ ), in parallel with decreased participation by repeat donors with an interdonation interval of 12 months or less (from $10 \%$ to $<1 \%$ ), might have affected the overall HIV and HCV incidence rates reported in Tables $\mathbf{1}$ and $\mathbf{2}$. However, these factors did not change the incidence of viral conversion (Table 3) since the last screen-negative donation or the corresponding residual risk estimates because co-infection with another infectious agent (mainly HBV) screened them out anyway. The interaction of these factors is complex. The vast majority of publications have reported a higher prevalence of blood-borne infections in first-time donors compared with repeat donors, including in Brazil ${ }^{1,5-9,10}$, thus implying higher disease incidence as well. However, the present study showed evidence to the contrary, with HIV and HCV prevalences being approximately three- and two-fold higher, respectively, in the repeat donors compared with the first-time donors. The increase in HIV prevalence was much higher among male donors of 16-24 years of age in comparison with any other demographic segment (Figures 1 and 2), reinforcing the hypothesis of test-seeking behavior among the former. Independent evidence of such behavior was obtained in the State of São Paulo, where 9\% of all donors were estimated to be HIV test seekers with a high risk for sexually transmitted diseases ${ }^{10,13}$. In the State of Santa Catarina, a sudden rise in HIV prevalence among blood donors was observed in the beginning of the 2000s after a consistent downward trend in the 1990s, possibly associated with HIV test seeking? The HIV incidence per thousand repeat blood donors per year increased from 0.36 (CI 95\% 0.13-0.79) at the end of the $1990 \mathrm{~s}^{5}$ to $0.86(0.73-1.00)$ during the $2007-2013$ period, as shown in the present study. This worrying result requires a rethinking of current prevention strategies and immediate action to reduce HIV transmission among the blood donor population.

It is important to recall that that the number of converting repeat donors is typically lower for the incidence rate of viral conversion used for the residual risk calculation because of exclusion of HIV-positive donors using other screen-positive test results. The latter are mainly markers of sexually transmitted 
TABLE 3 - HIV and HCV residual risk by donor type before and after implementation of NAT screening.

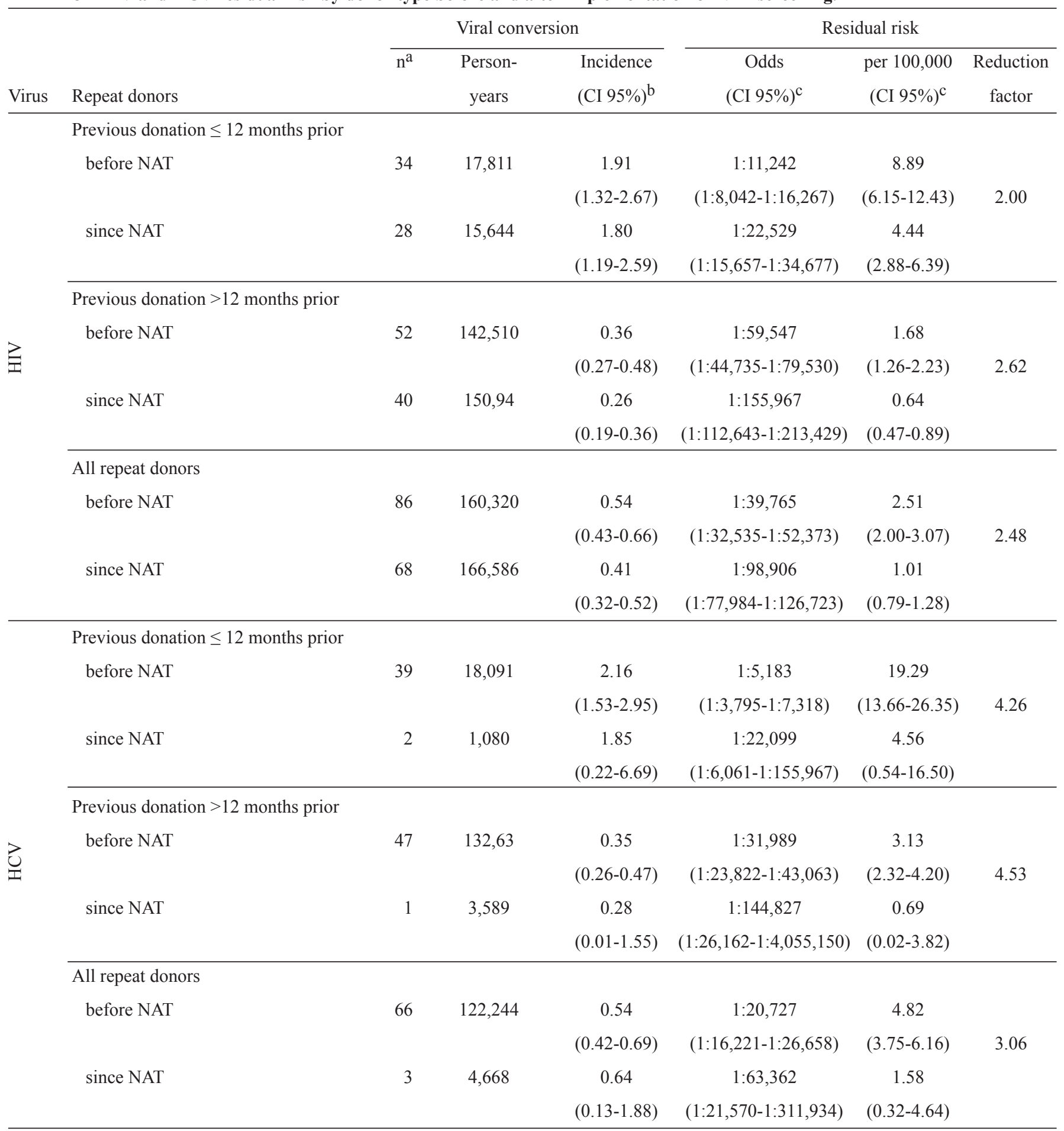

${ }^{\mathbf{a}}$ Number of converting donors without another screen-positive test result; ${ }^{\mathbf{b}}$ Viral conversion incidence per thousand person-years; ${ }^{\mathbf{c}_{\text {Residual }}}$ risk and 95\% CI, assuming an HIV immunological window period of 17 or 9 days for before or since NAT implementation, respectively, and an HCV window period of 32.6 and 9 days for before or since NAT implementation, respectively. HIV: human immunodeficiency virus; HCV: hepatitis C virus; NAT: nucleic acid amplification test.

diseases such as hepatitis B and syphilis, which indicate risky sexual behavior. The exclusion of these blood donors reduced the HIV incidence per thousand repeat donors per year from 6.25 (Table 1) to 1.91 and 1.81 (Table 3) for the periods before and after NAT implementation, respectively. As a consequence, the HIV incidence used for the residual risk calculation decreased slightly over the compared periods despite a considerable increase of HIV prevalence in young male donors over 
the same time period. Taken together, these findings suggest that halving the HIV residual risk after the implementation of NAT (Table 3) was mainly due to a reduction in the immunological window period rather than a reduction in the incidence of HIV conversion. Among the repeat donors for whom the previous inter-donation interval was longer than 12 months, HIV residual risk was reduced 2.62-fold during the NAT screening period.

Overall, the HCV incidence in HEMOSC was halved after the NAT implementation (Table 2). Although this change did not reach statistical significance, it contributed to a more than four-fold reduction in the HCV residual risk in repeat donors, suggesting that the downward trend reported in the 1990s, during which the HCV residual risk decreased to 51 per 100,000 repeat donors per year ${ }^{6}$, is continuing. The present study showed a further reduction of this risk to an approximately five-fold lower level. However, HCV incidence was approximately twenty times higher in the repeat donors whose last inter-donation interval was shorter than 12 months compared with those having longer intervals. Despite relatively rare sexual transmission of $\mathrm{HCV}$, this infection is still a marker of risky behavior for various blood-borne diseases. Therefore, the magnitude of the above difference in $\mathrm{HCV}$ incidence rates reinforces the hypothesis of HIV test seeking among some repeat donors with shorter inter-donation intervals.

The similarities in magnitude and direction of both prevalence and incidence regarding the risk factors analyzed underline the notion of prevalence as a cumulative incidence and its use for estimating the viral conversion risk in first-time donors ${ }^{14}$. In Brazil, higher HIV prevalence rates among men and those with lower educational levels were reported for both the general population ${ }^{15}$ and blood donors ${ }^{10}$, including in the capital of Florianópolis compared with other regions of the State of Santa Catarina ${ }^{1,16}$. The present study showed that the City of Criciúma had the highest HIV and HCV risk among blood donors. An HCV incidence of 3.11 per 100,000 donor-years and an HCV residual risk of 0.5 per 100,000 were reported for the capitals of the federal States of São Paulo, Rio de Janeiro and Minas Gerais in 2007, along with a downward trend over time ${ }^{17}$. This finding is in line with similar trends among HEMOSC blood donors.

Increases in the overall HIV and HCV incidence rates during the NAT screening period may be a consequence of higher NAT sensitivity compared with serological testing. For HIV, a high prevalence of 1.46 per thousand donors in the present study was close to the prevalence of 1.51 reported in South Africa but is considerably higher than the prevalence rates of 0.23 reported in the Mediterranean and Central Europe and 0.34 reported in Southeast Asia ${ }^{18}$.

This paper is the first report on the performance of Brazilian $\mathrm{HIV} / \mathrm{HCV}$ NAT screening on prevalence and incidence in blood donors. An HIV residual risk of 2.51 per 100,000 per year observed in HEMOSC between 2007 and 2013 is close to the value of 2 per 100,000 per year reported at the turn of the century in the Florianópolis metropolitan $\operatorname{area}^{5,7}$ and in the City of Lages ${ }^{9}$ but lower than the 3.82 estimated for the entire State $^{1}$. The data compiled from the blood banks of São Paulo, Belo Horizonte and Recife during the 2007-2008 period produced an HIV residual risk of 1.13 per 100,000 and projected its reduction by a factor of 1.66 when using six-sample mini- pools and an immunologic window duration of nine days, or by a factor of 2.69 when using individual NAT screening and a window period of 5.6 days ${ }^{10}$. An HIV residual risk of 2.51 per 100,000 in HEMOSC and its reduction by a factor of 2.49 when using six-sample mini-pools for NAT screening are reasonably close to the aforementioned projections.

Among the present study limitations, it is worth mentioning the lack of publications on the Brazilian NAT kit's limits of detection and the focus on residual risk. The latter is the risk of transfusing an infected blood unit; however, the risk of a recipient becoming infected with the transfusion-transmitted agent still depends on the minimal infectious dose and the host immunological response. Recent studies have emphasized the risk of a recipient developing a transfusion-transmitted infection $^{18,19}$, which will also be estimated for the Brazilian NAT data in the near future. Another limitation is the low precision of rare events, such as viral conversion, thus limiting the comparisons across regions and/or countries. Finally, although the so-called NAT yield (the proportion of serologically negative and NAT-positive cases) is a direct measure of the NAT's impact on blood safety, the only way to compare the impact before versus after NAT implementation is to use the incidence/window period model that is available for both periods.

Despite large reductions in HIV and HCV residual risk after the implementation of NAT screening in the State of Santa Catarina, the overall HIV incidence of over one per thousand donor-years is almost one hundred times higher compared with countries such as France, Germany, the USA, Canada and Australia ${ }^{20-26}$. More effective prevention measures need to be implemented in the Brazilian general population before transfusion risk can be reduced further ${ }^{1}$. Otherwise, technological advances, such as individual NAT screening, will still have a limited beneficial impact on blood safety. Therefore, better integration between blood safety policies and policies that prevent sexually transmitted diseases in the general population should dominate the research and development agenda in this area.

\section{ACKNOWLEDGMENTS}

The authors would like to thank the working group on blood and hemocomponents from the Brazilian Ministry of Health and the FIOCRUZ scientists who developed the HIV/HCV NAT kit for their insightful comments.

\section{CONFLICT OF INTEREST}

The authors declare that there is no conflict of interest.

\section{FINANCIAL SUPPORT}

The work was supported by joint funding from the Brazilian Ministry of Health and FAPESC (Fundação de Apoio à Pesquisa Científica e Tecnológica do Estado de Santa Catarina) via grant number 15.973/2009-5. 


\section{REFERENCES}

1. Maresch C, Schluter PJ, Wilson AD, Sleigh A. Residual infectious disease risk in screened blood transfusion from a high-prevalence population: Santa Catarina, Brazil. Transfusion 2008; 48:273-281.

2. Roth WK, Busch MP, Schuller A, Ismay S, Cheng A, Seed CR, et al. International survey on NAT testing of blood donations: expanding implementation and yield from 1999 to 2009. Vox Sang 2012; 102:82-90.

3. Canutti Júnior V. Risco transfusional: Metodologia e estudo. Atualização em Hemoterapia 1998; 5:90-99.

4. Covas DT. Risco de transmissão do HIV-1 pelas transfusões de sangue. Atualização em Hemoterapia 1998; 5:100-106.

5. Kupek EJ. Residual transfusion risk for hepatitis B and C in southern Brazil, 1991-1999. J Viral Hepat 2001; 8:89-82.

6. Kupek EJ. The redution of HIV reduction of HIV transfusion risk in southern Brazil in the 1990s. Transfusion Med 2001; 11:75-78.

7. Kupek E. Transfusion risk for hepatitis B, hepatitis C and HIV in the state of Santa Catarina, Brazil, 1991-2001. Braz J Infect Dis 2004; 8:236-240.

8. Barreto CC, Sabino EC, Gonçales TT, Laycock ME, Pappalardo BL, Salles NA, et al. Prevalence, incidence, and residual risk of human immunodeficiency virus community and replacement first-time blood donors in São Paulo, Brazil. Transfusion 2005; 45:1709-1714.

9. Spada C, Souza MA, Treitinger A. Estimation of the Residual Risk for the Transmission of HIV in Blood Donors from the Mountain region of Santa Catarina. Braz J Infect Dis 2005; 9:489-493.

10. Sabino EC, Gonçalez TT, Carneiro-Proietti AB, Sarr M, Ferreira JE, Sampaio DA, et al. Human immunodeficiency virus prevalence, incidence, and residual risk of transmission by transfusions at Retrovirus Epidemiology Donor Study-II blood centers in Brazil. Transfusion 2012; 52:870-879.

11. Pinto AR, Petry A, Graef T, Vandresen R, Kupek EJ. Case report of haemovigilance investigation using phylogenetic analysis of HIV-1 in Brazil. Transfus Med 2012; 22:57-62.

12. Schreiber GB, Busch MP, Kleinman SH, Korelitz JJ. The risk of transfusion-transmitted viral infections. NEJM 1996; 334:1685-1690.

13. Goncalez TT, Sabino EC, Murphy EL, Chen S, Chamone DA, McFarland W. Human immunodeficiency virus test-seeking motivation in blood donors, São Paulo, Brazil. Vox Sang 2006; 90:170-176.

14. Zou S, Fang CT, Dodd RY. A method for estimating incidence rate of infectious diseases among first-time blood doors. Transfusion 2008; 48:1827-1832.
15. Fonseca MG, Bastos FI. Twenty-five years of the AIDS epidemic in Brazil: principal epidemiological findings, 1980-2005. Cad Saude Publica 2007; 23 (supl III): S333-S344.

16. Rosini N, Mousse D, Spada C, Treitinger A. Seroprevalence of HbsAg, anti-HBc and anti-HCV in Southern Brazil, 1999-2001. Braz J Infect Dis 2003; 7:262-267.

17. Almeida-Neto C, Sabino EC, Liu J, Blatyta PF, Mendrone-Junior A, Salles NA, et al. Prevalence of serological markers for hepatitis B and C viruses in Brazilian blood donors and incidence and residual risk of transfusion transmission of hepatitis C virus. Transfusion 2013; 53:827-834.

18. Bruhn R, Lelie N, Custer B, Busch M, Kleinman S, International NAT Study Group. Prevalence of human immunodeficiency virus RNA and antibody in first-time, lapsed and repeat blood donations across five international regions and relative efficacy of alternative screening scenarios. Transfusion 2013; 53:2399-2412.

19. Vermeulen M, Lelie N, Sykes W, Crookes R, Swanevelder J, Gaggia L, et al. Impact of individual-donation nucleic acid testing on risk of human immunodeficiency virus, hepatitis B virus, and hepatitis $C$ virus transmission by blood transfusion in South Africa. Transfusion 2009; 49:1115-1125.

20. Glynn SA, Kleinman SH, Schreiber GB, Busch MP, Wright DJ, Smith JW, et al. Trends in incidence and prevalence of major transfusiontransmissible viral infections in US blood donors, 1991 to 1996. Retrovirus Epidemiology Donor Study (REDS). JAMA 2000; 284:229-235.

21. Dodd RY, Notari EP, Stramer SL. Current prevalence and incidence of infectious disease markers and estimated window-period risk in the American Red Cross blood donor population. Transfusion 2002; 42:975-979.

22. Chiavetta JA, Escobar M, Newman A, He Y, Driezen P, Deeks S, et al. Incidence and estimated rates of residual risk for HIV, hepatitis C, hepatitis B and human T-cell lymphotropic viruses in blood donors in Canada, 1990-2000. CMAJ 2003; 169:767-773.

23. Seed CR, Kiely P, Keller AJ. Residual risk of transfusion transmitted human immunodeficiency virus, hepatitis $B$ virus, hepatitis $C$ virus and human T lymphotrophic virus. Intern Med J 2005; 35:592-598.

24. Busch MP, Glynn SA, Stramer SL, Strong DM, Caglioti S, Wright DJ, et al. A new strategy for estimating risks of transfusion-transmitted viral infections based on rates of detection of recently infected donors. Transfusion 2005; 45:254-264.

25. Pillonel J, Le Marrec N, Girault A, David D, Laperche S. Epidemiological surveillance of blood donors and residual risk of blood-borne infections in France, 2001 to 2003. Transfus Clin Biol 2005;12:239-246.

26. Zou S, Dorsey KA, Notari EP, Foster GA, Krysztof DE, Musavi F, et al. Prevalence, incidence, and residual risk of human immunodeficiency virus and hepatitis $\mathrm{C}$ virus infections among United States blood donors since the introduction of nucleic acid testing. Transfusion 2010; 50:1495-1504. 